\title{
Collaborative Governance on Public Security in Concentrated Contiguous Destitute Areas: A Case Study of Wuling Mountains Area in China
}

\author{
Anhua Yang \\ School of Politics and Law, Jiangsu Normal University, Xuzhou, China \\ Email: anhua7321@163.com \\ Received 24 March 2015; accepted 3 May 2015; published 8 May 2015 \\ Copyright (C) 2015 by author and Scientific Research Publishing Inc. \\ This work is licensed under the Creative Commons Attribution International License (CC BY). \\ http://creativecommons.org/licenses/by/4.0/

(c) (i) Open Access

\begin{abstract}
In 2011, China initiated the strategy of Regional Development and Priority Poverty Alleviation in Concentrated Contiguous Destitute Areas. The vital method as well as the goal of this strategy is to revolutionize current special difficult situation by collaborative development of regional integration and basically realize the goal of building a well-off society in an all-around way synchronizing with the country. The poor condition of public security is the fundamental factor of constricting the development of Chinese contiguous destitute areas, thus leading to its lagged development. Collaborative governance of public security is not only the most basic component of regional integration but also a fundamental requirement of improving the security condition in contiguous areas for the successful implementing of the strategy mentioned above. This paper points out a framework by mainly focusing on Wuling Mountains areas in China. The system consists of conventional and unconventional public security as its content, as well as "the collaboration of public security" and "the cooperation among regional zone" as its structure.
\end{abstract}

\section{Keywords}

Collaborative Governance, Public Security, Contiguous Destitute Areas, Wuling Mountains Area

\section{Introduction}

Wuling Mountains Area, centering on Wuling Mountains, neighbors on Human-Huber-Qingdao-Guangzhou areas, and includes 71 counties, cities and regions among the four provinces with a total area of 171.8 thousand 
square kilometers. At the end of 2010, the area had a total population of 36.45 million, including 27.92 million peasants. Wuling Mountains Area integrates old revolutionary areas, regional areas and poor areas as a whole. It is regarded as a contiguous destitute area that has a long cross-province line, many ethnic minorities and wide distribution of impoverished people.

Since 2011, China has initiated the national new strategy-Regional Development and Priority Poverty Alleviation in Concentrated Contiguous Destitute Areas, which classified 14 regions including Wuling Mountains area, Wumeng Mountains area and Qinba Mountains area into concentrated contiguous destitute areas ("contiguous areas” or "contiguous destitute areas" hereafter), and regarded them as main battleground of priority poverty alleviation in the following 10 years. This strategy is different from Chinese previous regional development and poverty alleviation plan in that it regarded "drive poverty alleviation by regional development; poverty alleviation facilitate regional development” as basic ideas. Therefore, it would be a brand-new mode of development that organically combines regional development and poverty alleviation and changes the traditional mode of no relevance between them at all [1]. The implement of this strategy is significant to the realization of national general strategy, building a well-off society in an all-around way and even the magnificent goal of socialist modernization. Moreover, it also points out a series of brand-new projects for the academic field. This article mainly studies Wuling Mountains Area to give a tentative discussion about collaborative governance on public security, hoping to broach the subject.

The important method and goal of contiguous destitute areas regional development and priority poverty alleviation is "sending regional development into the track of collaborative development by establishing an overall regional cooperation mechanism, thus revolutionizing current special difficult situation and basically realizing the goal of building a well-off society in an all-around way synchronizing with the country” [2]. There are lots of questions to be studied since it is arduous to realize the goal in such special areas where the economy is extremely poor. Facing so many questions and conflicts, we need to analyze the contradiction so as to seize the major one. The poor condition of public security is the fundamental factor of constricting the development of Chinese contiguous destitute areas, thus leading to its lagged development. Revolutionizing public security condition is not only the start point of the development of contiguous destitute areas, but also the prerequisite for the development of contiguous destitute areas and Priority Poverty Alleviation Strategy. What's more, the implement of this strategy must start with collaborative governance on public security.

This is determined by the goal of contiguous destitute areas development as well as its special condition. The important goal of this strategy is "sending regional development into the track of collaborative development by establishing an overall regional cooperation mechanism” [3]. The collaborative development of contiguous areas is not only the important goal of the strategy, but also its key method. It is the biggest difference between this strategy and Chinese previous development strategies. However, the first step to realize regional integration is to realize the collaborative governance on public security within the contiguous areas. The reason is, collaborative governance on public security is not only the basic component of regional integration but also a method to fundamentally improve the condition of public security and provide security assurance for the economic development in contiguous areas and the smooth implementation of the strategy, which is the prerequisite for the development of contiguous destitute areas and Priority Poverty Alleviation Strategy as well as the fundamental requirement of securing the smooth implementation of the strategy.

\section{Literature Review}

\subsection{The Study on Public Security Governance}

The study on trans-boundary crisis and its governance. As the characteristic of trans-boundary becomes increasingly prominent, trans-boundary crisis becomes the frontier field of crisis research in recent years. Based on the trans-boundary characteristics and knock-on results of modern crisis, Scholars (e.g. Boin, 2009) carried out variant degree research on the concept, feature, evolution of trans-boundary crisis and the corresponding governance tactics [4]. Zhu Zhengwei, Jin Taijun, Zhong Kaibin, Ma Ben, Lv Zhikui, Zhang Haibo and Xi Bao also provide their analysis towards trans-boundary crisis from different point of view. All these researches highlight the trans-boundary collaborative governance of modern crisis. Zhang further analyzes the new challenges that trans-boundary crisis brought to public administrators [5].

The study on regional public crisis and public security governance. At present, frequently occurrence of regional public issues highlights the contradiction between the governance mode of traditional introverted bureau- 
cracy administration as well as unilateral administration and the externality of regional public issues. How to break the restriction of traditional bureaucracy administration and administrative division for the purpose of govern public security issues more effectively as a basic public business in a certain region has become the new focus of emergency management study both home and abroad. Boin and Rhinard pointed out the scheme for EU public security capacity construction on the basis of analyzing the challenges crisis brought to EU [6]. Chen talked about the establishment of the coping mechanism of regional disaster and risk interaction from the angel of severe disaster and risk [7]. Teng pointed out the specific model of regional crisis emergency interaction [8]. Yang believed we should adopt corresponding local cooperation mechanisms according to the crisis in different fields in order to deal with regional public crisis [9]. Shi put forward regional integrated public security administrative model [10]. Xia even systematically discussed the theory and model of public security management [11].

The study on collaborative governance on public security. Recently, synergistics is applied into the public security governance study aiming at the fact that the whole group collaboration is hard to achieve because of departmental and regional barriers in Chinese public security governance. Zhang considered that we should improve public security governance capacity by the collaborative governance between government and the public [12]. Liu talked about strategy and specific method of public security governance fragments arrangement in local government using integrity governance as a theoretical tool [13]. Fan pointed out that we should establish public security collaborative coping mechanisms that involves many sectors, many levels, and many processes [14]. Zhang applies collaboratives principle to carry out a tentative research on the construction of public crisis collaborative governance [15]. Sha believed that the establishment of collaborative governance structure and mechanism was the main method of public crisis collaborative governance [16].

\subsection{The Study on the Issue of Contiguous Destitute Areas Development}

The proposal of the concept of “concentrated contiguous destitute areas”. China usually adopts the concept of "concentrated contiguous special type” district when it comes to priority poverty alleviation. The concept of “concentrated contiguous destitute areas" was formally put forward for the first time by Premier Wen Jiabao in July, 2010. The state's “twelfth five-year” development plan pointed out “moving rapidly to address the poverty of concentrated contiguous destitute areas”. National Program for Rural Poverty Alleviation (2001-2010), focusing on the goal of comprehensive constructing well-being society, adjust the previous 18 concentrated destitute areas into 14 "concentrated contiguous destitute areas" and formally pointed out the national strategy of making “concentrated contiguous destitute areas” as the main battlefield of priority poverty alleviation in the future.

The study on the development of contiguous destitute areas. With the brewing and the formulation of regional development and Priority Poverty Alleviation Strategy, some scholars set about studying in this field. Huang analyzed the strategy of poverty alleviation and its development [17]. Cao measured poverty in destitute areas of specific type in China from different angels and pointed out the intervening measures [18]. Chen, mainly foucusing on Wuling Mountains Area, conducted multi-dimensional measurement towards the poverty in contiguous destitute areas [19]. Zhang talked about the relevance between disasters and poverty of concentrated contiguous ethnic minorities in arduous community [20]. Wang analyzed the collaborative method of regional development in Wuling Mountains Area [21]. Lv discussed the theoretical innovation of destitute governance in contiguous destitute areas [22]. Li analyzed the collaborative provision of public products in contiguous destitute areas [23]. Tong, Zeng and Jun analyzed the cultivation of growth poles in contiguous destitute areas [24].

From the above research, we can see that, in the aspect of public crisis and security governance, although the present research realize the necessity of trans-boundary governance and trans-boundary crisis even become the frontier field of international crisis study, the mature theoretical framework hasn't been formed. Moreover, present research mainly focuses on developed areas (an example is EU). In China, a small number of research started to pay attention to the issues of public security governance in developed areas like Yangtze River Delta and Pearl River Delta, but, in general, we still stay in the position of verifying western theories or using them to explain China's actual period. The fact is that localized theoretical frameworks that can be applied to Chinese actual condition are rare and they often neglect the study on public security and its governance in underdeveloped areas between provinces-contiguous destitute areas.

The academic field starts to focus on the study of contiguous destitute areas, but mainly in the filed of economic development and poverty alleviation, which leads to the neglect of public service especially the basic issue like public security. It has become an urgent task in academic field to strengthen the study on collaborative 
governance of public security in contiguous areas and put forward a public security governance strategy to guide practice in order to provide security for the successful implementation of this strategy as well as to lay the foundation and create sound environment for integrative development in contiguous areas.

\section{Study Content and Framework}

\subsection{Theoretical Interpretation and Framework Construction}

The choice of theory and the establishment of research framework is the basis of taking on scientific research. This kind of research need to: put forward the analytical concept of boundary conflict, boundary balance, and boundary co-existence thus formulating a concept system of boundary in organization structural analysis, by analyzing boundary and the influence brought by its "barrier effect" and "intermediary effect"; analyze the essentials and principles of synergistics as well as the compatibility between it and the Chinese trans-boundary public security governance; to define the concept, conclude the features, and adopt the analytic hierarchy process method when it comes to intergovernmental management, governance theory classification and collaborative governance on public security; formulate a framework that intermingle "public security collaboration" with "cooperation between administrative region” on the above basis. To conclude, on one hand, we should establish public security collaborative framework system by two subsystem, conventional and unconventional public security. On the other hand, in the field of administrative division, we should establish a regional trans-boundary system consists of 4 province-level, 11 city-level and 71 county-level subsystem.

\subsection{The Particularity of Public Security in Contiguous Areas and the Barrier Analysis of Collaborative Governance on Public Security}

Particular condition of an area determined the particularity of the public security condition as well as lots of barriers of collaborative governance on public security in the area. Deep analysis of these issues is the start point of the study in this field. First, we need to construct risk assessment model to evaluate the existing risk and crisis of Wuling Mountains Area, which includes risk evaluation, conclusion of risk type, and the comprehensive evaluation of the occurrence, evolution, derivation of crisis and its effect to both regional economic society and risk governance. Second, we should analyze systematically the evolution mechanism of risks in contiguous areas. Moreover, we should also further study the existing and the potential problems of public security governance in contiguous areas from the perspective of structural defects and institutional shortcomings, thus analyzing the social "relationship chain" between interested parties and its profound influence to trans-boundary (mainly in administrative level) public security governance under the framework of "conflict-cooperation-order" and the double restraints-resources scarcity and the rationality of “economic man”.

\subsection{The Collaborative Governance of the Crime Prevention and Control System and Mechanism in Contiguous Areas}

The establishment of a sound crime prevention and control system and mechanism is the basis of realizing collaborative governance on public security in contiguous areas. This part mainly includes: constructing the concept of collaborative police in contiguous areas; establishing an overall crime prevention and control system consisting of patrols, security and prevention and control network; establishing trans-boundary collaborative service command system, image monitoring system, integrated information system, communication network system and GPS system in contiguous areas. Based on this, we will finally break the social security collaboration system and operational mechanism in cross-basin administrative region.

\subsection{The Collaborative Governance of the Ecological Security System and Mechanism in Contiguous Areas}

The collaborative governance of the ecological security can protect the ecological environment, improve people's livelihood vulnerability, and ensure economic and social sustainable development in contiguous areas. Therefore, it is not only the inevitable choice for people to eventually get rid of poverty, but also an important part of collaborative governance on public security in contiguous areas. We should study: trans-boundary settings and collaborative operation of the environmental protection agency; intergovernmental collaborative go- 
vernance of cross-basin water pollution; precaution and emergency of the ecology risk, interest compensation and sharing mechanism, and the collaborative governance of the ecological security system and mechanism that integrates ecological environment protection laws, social participation and supervision mechanism.

\subsection{The Construction of Collaborative Governance System for Significant Emergent Public Crisis Events in Contiguous Areas and Its Institution Design}

Compared to the former two aspects of conventional public safety, this is an unconventional collaborative governance on public security that surpasses administrative divisions, highlights regional collaboration and integrity, aiming to govern significant emergent public security events in contiguous areas. It should include the design of the institutional framework in three aspects: institutionalized collaboration protocol, network governance and overall operation mechanism or process [4]. It also require specific studies: the establishment of a unified and collaborated emergency institutions in contiguous areas; the formulation of rules and regulations for collaborative governance on public crisis; the formulation of emergency preparedness plan; the establishment of information platform of collaborative governance (including application networks, databases, GIS, etc., and establishing required database for public security governance in contiguous areas); sharing mechanism of the infrastructure in collaborative governance; the establishment of resource safeguard mechanism of collaborative governance; the power-responsibility system partition in each administrative government (and its departments); collaborative governance on culture and the cultivation of social capital. We can thereby form a public security work system consisting of institutions, mechanisms, personnel and technology, while, by collaboration protocol, network administrative institutions, and overall operation mechanisms or processes, making the executive body of contiguous areas promotes cross-regional, inter-departmental inter-organizational collaborative action relying on collaborative networks. It will promote trans-regional collaborative action by collaborative network in contiguous areas, thus achieving achieving a collaborative action with resource integration and sharing of public security governance across provinces and cities at the core.

\section{Conclusion}

Regional development in contiguous destitute areas and priority poverty alleviation are vital adjustment of Chinese development strategy in the new period. The smooth implementation of them requires not only practice and exploration but also theoretical innovation. The crisis management research from the perspective of social science in China has just started, but entered into the bottleneck period after it [25]. The important reason for this is the lack of sufficient concern and refining of Chinese issues. Therefore, one of the basic ways for researchers to get out of the bottleneck is basing themselves on Chinese reality and refining the unique project of China's crisis management while fully grasping the pulse of China's economic and social development. Nowadays, transboundary crisis research has become the forefront field of international crisis management research. It is our bounden duty to give proper response to this field and promote crisis management research while solving practical problems in China's crisis management, thus making Chinese crisis management establish its own discourse system in the international academia. The formulation of regional development in contiguous destitute areas and Priority Poverty Alleviation Strategy provides a valuable chance for us. The deep study on collaborative governance on public security in contiguous destitute areas provides intellectual support for the successful implementation of national strategy. It is the important historical mission in the academic world of risk management. Meanwhile, by referring to our study, researchers in China also need to take this opportunity to establish a complete scientific research framework system in this field so as to provide a Chinese version for international trans-boundary crisis researches.

\section{Acknowledgements}

This paper is supported by the Ministry of Education Project (Grant No. 12YJAZH059), China Postdoctoral Special Fund (Grant No. 2014T70507), National Social Science Foundation of China (Grant No. 13CGL104) and Jungian Province Department of Education Project (Grant No. 2011SJB630057).

\section{References}

[1] Yang, A.H. (2012) Strategic Significance on Regional Development and Poverty Alleviation of Contiguous Destitute 
Areas in China. People’s Daily, 2012-5-17.

[2] The State Council Poverty Alleviation Office (2011) The Strategy for Regional Development and Poverty-Alleviation in Wuling Mountain Areas (2011-2020).

[3] The State Council Poverty Alleviation Office (2011) The Outline of China's Development-Oriented Poverty Relief for the Rural Areas (2011-2020).

[4] Boin, A. (2009) The New World of Crises and Crisis Management: Implications for Policymaking and Research. Review of Policy Research, 26, 367-377. http://dx.doi.org/10.1111/j.1541-1338.2009.00389.x

[5] Zhang, W. (2011) An Analysis of Cross-Border Crisis and the Challenges for Administrators. Journal of Jishou University (Social Sciences Edition), 25, 72-76.

[6] Boin, A. and Rhinard, M. (2008) Managing Transboundary Crises: What Role for the European Union? International Studies Review, 10, 1-26. http://dx.doi.org/10.1111/j.1468-2486.2008.00745.x

[7] Chen, B. (2008) On the Establishment of a Regional Linkage System—Based on the Visual Threshold of Serious Disasters and Risks. Journal of Jishou University (Social Sciences Edition), 23, 133-137.

[8] Teng, W.X. (2010) Research on Regionally Integrated Emergency Response System. Social Sciences, 53, 63-68.

[9] Yang, L. (2011) The Role of Local Cooperation in Solving Regional Public Crisis Management. Wuhan University Journal (Philosophy and Social Sciences), 61, 57-68.

[10] Shi, P.J. (2006) Integrated Regional Public Security Management Mode and Strategies of China. Journal of Natural Disasters, 36, 9-16.

[11] Xia, B.C. (2011) Introduction to Public Safety Management. Contemporary China Publishing House, Beijing.

[12] Zhang, H.M. (2007) The Collaborative Response: Public Participation in Public Crisis Management. Changbai Journal, 23, 68-71.

[13] Liu, C. (2009) The Reorganization of the Fragmented Governance on Local Public Crisis. Journal of Jishou University (Social Sciences Edition), 16, 78-81.

[14] Fan, W.D. (2007) Advisement and Suggestion to Scientific Problems of Emergency Management for Public Incidents. Bulletin of National Natural Science Foundation of China, 12, 71-76.

[15] Zhang, L.R. (2007) Synergy-Governance and Innovation of Public Crisis Management: A Perspective of Synergetics. Chinese Public Administration, 62, 99-104.

[16] Sha, Y.Z. (2010) On Synergy Governance of Public Crisis. Chinese Public Administration, 25, 73-77.

[17] Huang, C.W. (2009) Poverty Reduction and Development Strategy of Concentrated Contiguous Areas with Particular Difficulties in China. Poverty Eradication, 4, 6-15.

[18] Cao, H.M. (2011) The Multidimensional Poverty Measure and Intervention in the Areas with Particular Difficulties. China Agriculture Press, Beijing.

[19] Chen, Q. (2012) The Multidimensional Measurement and the Policy Implication of the Rural Poverty in the Poverty-Stricken Covered Areas. Journal of Sichuan Normal University (Social Sciences Edition), 22, 58-63.

[20] Zhang, D.W. (2011) Association Study of Disaster and Poverty in Concentrated Minority and Community. Inner Mongolia Social Sciences, 35, 127-143.

[21] Wang, Z.Z. (2012) On the Collaboration Path for the Regional Development of the Wuling Area in China. Journal of Jishou University (Social Sciences Edition), 26, 134-140.

[22] Lv, F. (2012) Imagination of Development: Theoretical Innovation to Destitute Areas of Poor Governance. Journal of the Provincial Level Party School of CPC Sichuan Province Committee, 33, 112-117.

[23] Li, L.W. (2011) Regional Collaborative Supply of Public Goods: A New Idea in the Western Poverty Stricken Areas of Anti-Poverty. Issues in Agricultural Economy, 42, 91-96.

[24] Tong, Z.X., Zeng, Q. and Jun, M. (2012) The Strategic Analysis of the Growth Pole Cultivation in Contiguous Destitute Areas of China: A Case Study of the Wuling Mountain Region. China Soft Science, 64, 85-96.

[25] Lv, X.L., Zhang, H.B. and Zhong, K.B. (2012) Crisis Research in China under the Perspective of Public Administration: Past Patterns, Current Trends and Future Directions. Journal of Public Management, 9, 112-121. 